\title{
Recurrence plot analyses suggest a novel reference system involved in newborn spontaneous movements
}

\author{
BIRTE AßMANN \\ Free University Berlin, Berlin, Germany \\ MARCO THIEL and MARIA C. ROMANO \\ University of Potsdam, Potsdam, Germany \\ and \\ CARSTEN NIEMITZ \\ Free University Berlin, Berlin, Germany
}

\begin{abstract}
The movements of newborns have been thoroughly studied in terms of reflexes, muscle synergies, leg coordination, and target-directed arm/hand movements. Since these approaches have concentrated mainly on separate accomplishments, there has remained a clear need for more integrated investigations. Here, we report an inquiry in which we explicitly concentrated on taking such a perspective and, additionally, were guided by the methodological concept of home base behavior, which Ilan Golani developed for studies of exploratory behavior in animals. Methods from nonlinear dynamics, such as symbolic dynamics and recurrence plot analyses of kinematic data received from audiovisual newborn recordings, yielded new insights into the spatial and temporal organization of limb movements. In the framework of home base behavior, our approach uncovered a novel reference system of spontaneous newborn movements.
\end{abstract}

In comparison with the mature, skilled actions of adults and older children, which are smooth and precise, the movements of newborn and young infants lack the smooth temporal and spatial integration of coordinated actions. Limbs, head, and trunk seem to move as random elements, without reference to each other. Peiper (1963) described this overall impression of disorganization by saying that these "mass movements" are "awkward and abrupt and follow each other without connection" (p. 254).

However, there are many examples, in which the actions of newborn infants show some elements of coordination, meaning that various muscle groups work together, rather than as independent elements (Niemitz, 1989). These include well-known newborn reflexes such as the Moro, grasp, palmar, and plantar reflexes (e.g., Barnes, Crutchfield, \& Heriza, 1978; Bartlett, 1997; Capute, Accardo, Vining, Rubenstein, \& Harryman, 1978), functional actions such as rooting, sucking, and swallowing (e.g., Craig \& Lee, 1999; Crook, 1979; Peiper, 1963), hand-mouth synergism (e.g., Korner \& Beason, 1972; Takaya, Yukuo,

This study was supported by the Berliner Programm zur Förderung der Chancengleichheit für Frauen in Forschung und Lehre and Vivantes Geburtsklinik Neukölln. Correspondence concerning this article should be addressed to B. Aßmann, Institute of Human Biology and Anthropology, Free University Berlin, Albrecht-Thaer-Weg 6, 14195 Berlin, Germany (e-mail: birte.aszmann@gmx.de).
Bos, \& Einspieler, 2003), reaching toward objects (e.g., Corbetta \& Thelen, 1996; von Hofsten, 1982), and the coordination of leg movements in spontaneous kicking (e.g., Angulo-Kinzler, Ulrich, \& Thelen, 2002; Niemitz, 2002; Thelen \& Fisher, 1983; Thelen, Skala, \& Kelso, 1987).

These results regarding the coordinative features in the motor behavior of newborns motivated a more integrated investigation of the coordination of the overall system. New methods from nonlinear dynamics, combined with inspiration from models of animal exploratory behavior, allow a new understanding of the intrinsic dynamics of the unintentional movements of human infants. To establish a common language between biologists, psychologists, and systems theory scientists, it is necessary to obtain a notation system of behavior that corresponds to the natural morphology of behavior and is informative, parsimonious, and of predictive value. Common movement patterns, which are masked by the "ordinary language" used in the terminology of biology and psychology, can be revealed by a suitable notation system (Eilam \& Golani, 1988; Golani, 1992). In accordance with the results of recurrence plot analyses (Eckmann, Kamphorst, \& Ruelle, 1987; Ott, 1993), we applied symbolic dynamics (beim Graben \& Kurths, 2003) in order to transform the kinematic data into a simple but informative form of notation. These methods from nonlinear dynamics revealed a reference system involved in the behavioral motor organization that shows parallels to a methodological concept developed by 
Ilan Golani to discuss rat exploratory behavior as a natural manifestation of spatial learning (Biegler \& Morris, 1996; Eilam \& Golani, 1989; Etienne, Maurer, \& Séguinot, 1996). When rats are placed in a novel environment, they typically establish a home base. This is the place in which they stay for the longest cumulative time and to which the number of visits is especially large (Eilam \& Golani, 1989).

In the case of spontaneous newborn movements, the home base was given by limb/body positions that alternated with special movements and seemed to be both the origin and the target of each movement. From this perspective, recurrence plot analyses of the newborn movements suggest a reference system that is reminiscent - according to the above-mentioned criteria — of home base behavior and provides a basis on which to discuss the role of a reference system in newborn spontaneous movements in terms of concepts of spatial learning. Our study was designed to test whether and how new methods and concepts from different research fields can come together and shed light on old topics and even suggests a reference system of static body/limb configurations indicating some kind of coordination in the spontaneous movements of newborns.

\section{METHOD}

\section{Subjects}

The subjects were 6 apparently normal, full-term infants, 3 boys and 3 girls. Two subjects, Children 2 and 3, were dizygotic twins. The subjects were recruited in a maternity clinic to participate in a mini-longitudinal study of the development of newborn spontaneous movements over 3 to 10 days, depending on how long the mothers stayed at the clinic.

\section{Procedure}

The design of these observations was a frame-by-frame movement microanalysis using videotape recording. To videotape the awake infants, the parents were asked to bring their infants to the laboratory at a time between two anticipated feedings. For kinematic data collection, we used videorecording with three cameras $(50 \mathrm{~Hz})$ in a volume calibrated with a calibration frame. The infants were undressed and allowed to move spontaneously in the supine position for $20 \mathrm{~min}$. There were no specific stimuli presented, nor was the spontaneous posture of the infants controlled.

\section{Videotape Selection}

For the kinematic data analyses, we chose videotapes of each of the 6 infants from between 5 and 20 min on two or three different days. Restrictions were that the sequences should show continuous motor activity without resting or crying intervals longer than $20 \mathrm{sec}$.

\section{Kinematic Data}

Movement kinematics were analyzed with the Ariel Performance Analysis System (APAS). Every 12 frames, the 2-D positions of movement-relevant joints were tracked by a mouse click on the screen in each perspective. The APAS software allows one to compute, by triangulation, a 3-D picture from multiple 2-D frames. Movement-relevant joints were the shoulders, elbows, hands, hips, knees, and feet. Joint angles for the shoulders, elbows, hips, and knees were calculated from the coordinate data. Since the joint angle displacements of the elbow and shoulder of one arm and those of the hip and knee of the same leg displayed a correlation of .8 (data not shown), the movements of each limb could be captured by the
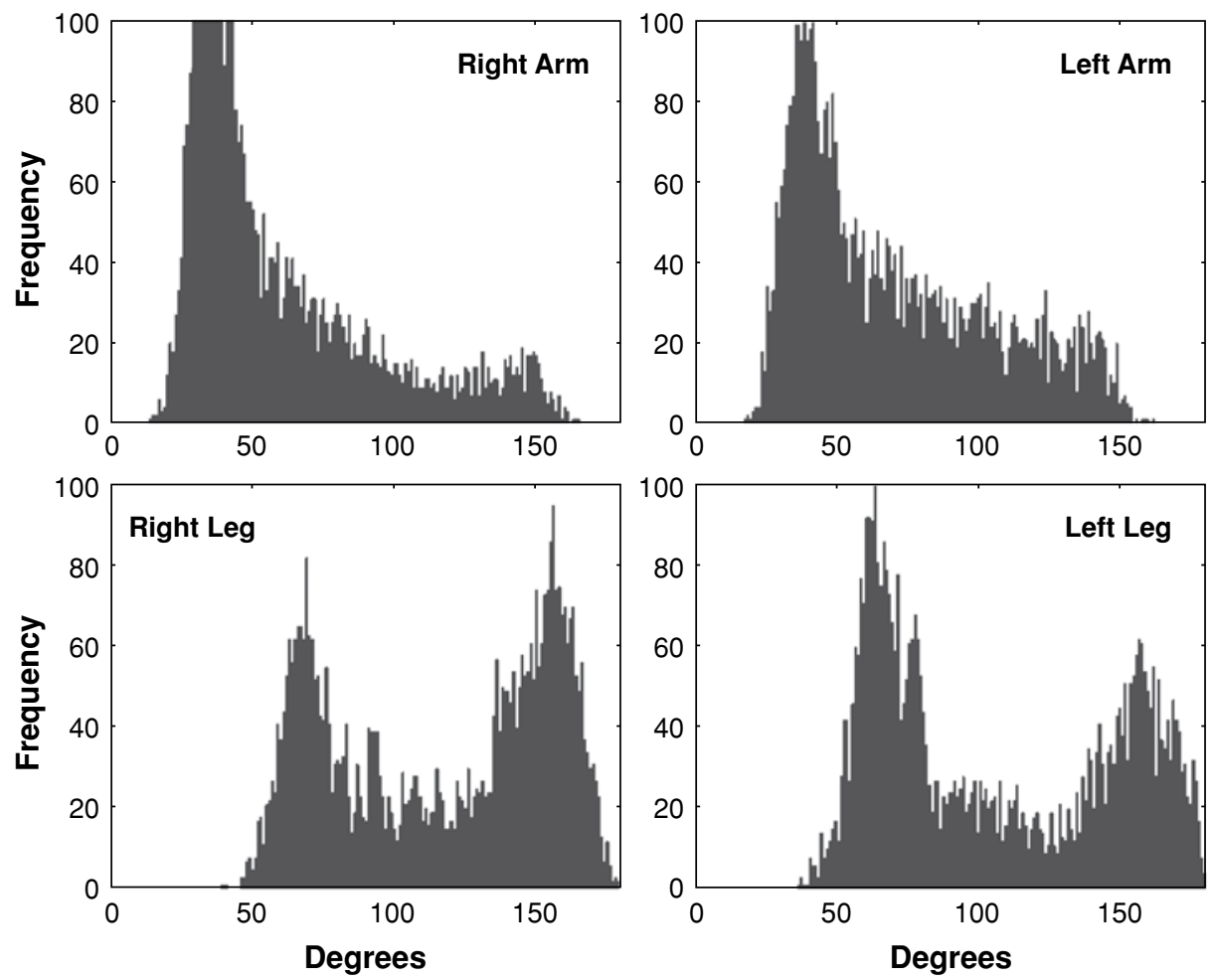

Figure 1. Frequency distribution of the joint angles of the single limbs for a 20-min movement episode. The frequency on the $y$-axis is plotted against the values of the joint angles on the $x$-axis. 
displacement of one angle. The arms were defined by the angle of the elbow, the legs by the knee angle.

\section{Symbolic Dynamics}

Symbolic dynamics is a natural way to describe data that appear as sequences of discrete states. This approach is based on a coarsegraining of the dynamics; that is, the time series are transformed into symbolic sequences by using very few symbols. This way, one loses some amount of detailed information, whereas some of the invariant, robust properties of the dynamics are kept (beim Graben $\&$ Kurths, 2003; Hao, 1991). To describe the body configurations of the newborns, we introduced a two-symbol encoding into the kinematic data. On the basis of the histograms in Figure 1, each limb was defined into two states: Either the limb was extended, or it was considered to be bent. For the arms, an elbow angle shorter than $60^{\circ}$ was regarded as angled, one higher than $60^{\circ}$ as stretched. For the legs, a knee angle shorter than $120^{\circ}$ was regarded as bent, one higher than $120^{\circ}$ as extended. From the combination of four angles with two possible states, there were 16 differently defined configurations.

\section{Recurrence Plot Analyses}

This method was first introduced to visualize the time-dependent behavior of a dynamical system, which can be represented as a trajectory $x_{i} \in \mathbf{R}^{n}(i=1, \ldots, N)$ in an $n$-dimensional vector space (Eckmann et al., 1987). It represents the recurrence of the trajectory in phase space to a certain state, which is a fundamental property of dynamical systems (Ott, 1993). The main step of this visualization is the calculation of the $N \times N$ matrix, $\mathbf{R}_{i, j}:=\Theta\left(\varepsilon-\left\|x_{i}-x_{j}\right\|\right)$, $i, j=1, \ldots, N$, where $\varepsilon$ is a cutoff distance, $\|\cdot\|$ is the maximum norm, and $\Theta(\cdot)$ is the Heaviside function. The binary values in $\mathbf{R}_{i, j}$ can be simply visualized as a matrix with the colors black (1) and white (0). The recurrence plot (RP) exhibits characteristic largescale and small-scale patterns that are related to typical dynamical behavior (Webber \& Zbilut, 1994). For a periodic signal of period $T$, the plot looks like Figure 2A for a very small $\varepsilon$. This is a series of stripes at $45^{\circ}$, with the stripes separated by a distance of $T$ in the vertical and horizontal directions. The RP of a chaotic system (Figure 2B) has a more complicated structure: (1) Due to the exponential divergence of nearby trajectories, the diagonal lines are interrupted, and (2) the distance between the diagonal lines is not constant, due to the multiple time scales present in chaotic systems. Brief episodes of parallel stripes at $45^{\circ}$ are hints of almost periodic trajectories. For the RP of white noise (Figure 2C), such a structure is not evident. It consists mainly of single points, indicating the randomness of the system. In all RPs, there is a stripe along the diagonal corresponding to $i=j$. Whereas the diagonals indicate a similar evolution of the different parts of the trajectory, the horizontal and vertical black lines show that the state of the system does not change for some time.
To capture the spontaneous movements of newborns, the configurational state of their body is defined by the four joint angles of the limbs. Four values of the angles belong to each sample point of the time series. For a sample point $i$, the vector $\mathbf{x}_{i}=\left[w_{1}(i), w_{2}(i)\right.$, $\left.w_{3}(i), w_{4}(i)\right]^{T}$ is generated, with $w_{1-4}(i)$ being the values of the four joint angles at sample point $i$. The same procedure is applied for the sample point $j: \mathbf{x}_{j}=\left[w_{1}(j), w_{2}(j), w_{3}(j), w_{4}(j)\right]^{T}$. These two vectors, which actually represent the same time series, depict the state of the system at sample points $i$ and $j$ for four angles. In the RP, the time series $\mathbf{x}_{i}$ on the horizontal axis and $\mathbf{x}_{j}$ on the vertical axis are compared with each other sample point by sample point. If the four values of the sample points $i$ and $j$ converge, there is a black dot, indicating that the state of the configuration in $\mathbf{x}_{i}$ and $\mathbf{x}_{j}$ are the same (according to the value of $\varepsilon$ ). If even one of the four values differs, there is a white dot, representing different configurations.

\section{RESULTS}

In our study, the spontaneous motor behavior of newborns was analyzed on the basis of the movements of the four limbs - that is, the arms and legs. For this purpose, we first focused on single limbs and then examined how this behavior was assembled into overall movement patterns. The following report will start with the results of symbolic dynamics and RP analyses referring to a 20-min movement sequence of 1 child.

\section{Motor Behavior of the Single Limbs}

The frequency distribution of the segment angles in which the limbs reside reveal one or two areas in which the limbs stay for a longer cumulative time than they do in other domains. Figure 1 shows, for the arms, a unimodal shape with a major peak in the lower range between $20^{\circ}$ and $60^{\circ}$; the legs show a bimodal shape with two equal peaks, one in the lower range between $50^{\circ}$ and $90^{\circ}$ and one in the higher range between $130^{\circ}$ and $170^{\circ}$. This distribution indicates that the arms are located predominantly in a bent position and relatively seldom in an extended position; the legs are located in a bent or extended position with comparable frequency, whereas the intermediate range $\left(90^{\circ}-130^{\circ}\right)$ is comparatively seldomly found. This means that the legs move mostly between the angled and the stretched positions and rest in these ranges and rarely on the way in between. The unimodal distribution of the
A

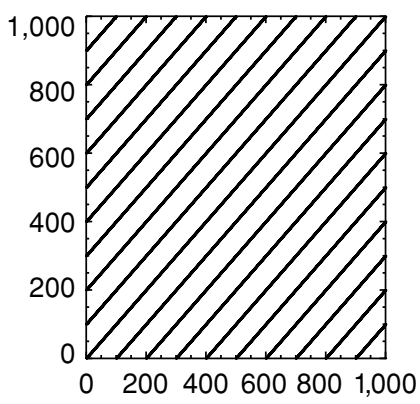

B

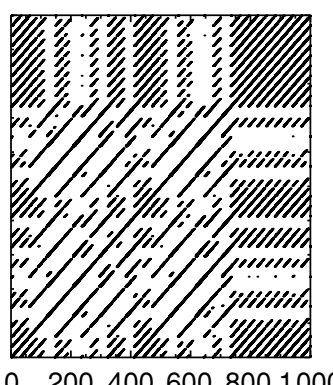

C

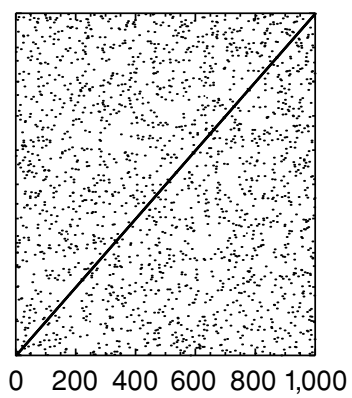

Figure 2. (A) Recurrence plot (RP) of a periodic function. (B) RP of a chaotic system (the Rössler system with standard parameters). (C) RP for random white noise. 
arms implies that the arms move mostly out of the bent position back to the bent position and exhibit only one resting position.

\section{Recurrence Plot Analysis}

From the combination of the joint angle positions of the four limbs at each sample point arise certain configurations in which the body resides. The dynamic of the distribution of these configurations can be visualized with an RP. Figure 3 presents the RP of the motor behavior of a newborn child, defined by the described configurations. The first thing we learn from the RP is that the movement organization is static, not dynamic states. This is shown by the rectangular structure of the RP on a time scale of 10-15 sample points $(3 \mathrm{sec})$. Such structure results from the black rectangles' being piled upon each other and interrupted by white rectangles. Black rectangles represent recurring configurations, and white rectangles represent other configurations.

If we look at the RP of single configurations by following a vertical line, we see that certain configurations have a high rate of recurrence, indicated by a high number of black dots. Other configurations occur rarely, indicated by a low number of black dots, seen as a white line. A closer look at single configurations reveals predominantly black sections in parts of the plot and white sections in other areas. This means that in parts of the time series, newborns use certain configurations very frequently and then, within the same time series, switch to other configurations.

The superior structure is demonstrated by the rectangular structure on a higher time scale of $400-500$ points of measurement. This pattern arises from particular configurations' recurring with a certain regularity in time windows of roughly $100 \mathrm{sec}$. On this time scale, alternations between different configurations become apparent and show that two to three configurations can serve as a reference system. Absent diagonal structures reveal that there are no recurring movement sequences or sequentially recurring successions of configurations.

Another measure of RPs is the length of vertical structures. The length of black vertical lines shows how long a particular configuration is held; the length of white vertical lines specifies the intervals between the recurrence of a configuration. Figure 4 exemplifies the frequency distribution of black vertical lines for two sample points and for white vertical lines for one sample point. The lengths of both the vertical black and the white lines do not display predominant values if calculated over the overall time series. This means that the time spans for which a configuration is maintained and the intervals of recurrence to this configuration are very variable.

\section{Frequency Distribution of the Configurations}

The RP demonstrates that decisive configurations are held very long and often, whereas others are held for a shorter duration and much less frequently. For further quantification, we introduced, on the basis of Figure 2 symbolic dynamics, so that each limb was defined by either a bent or an extended state. From this two-symbol coding of the four limbs resulted $4^{2}$ defined configurations. By the use of this technique, the studied movements can be described as follows: Limbs were moved singularly

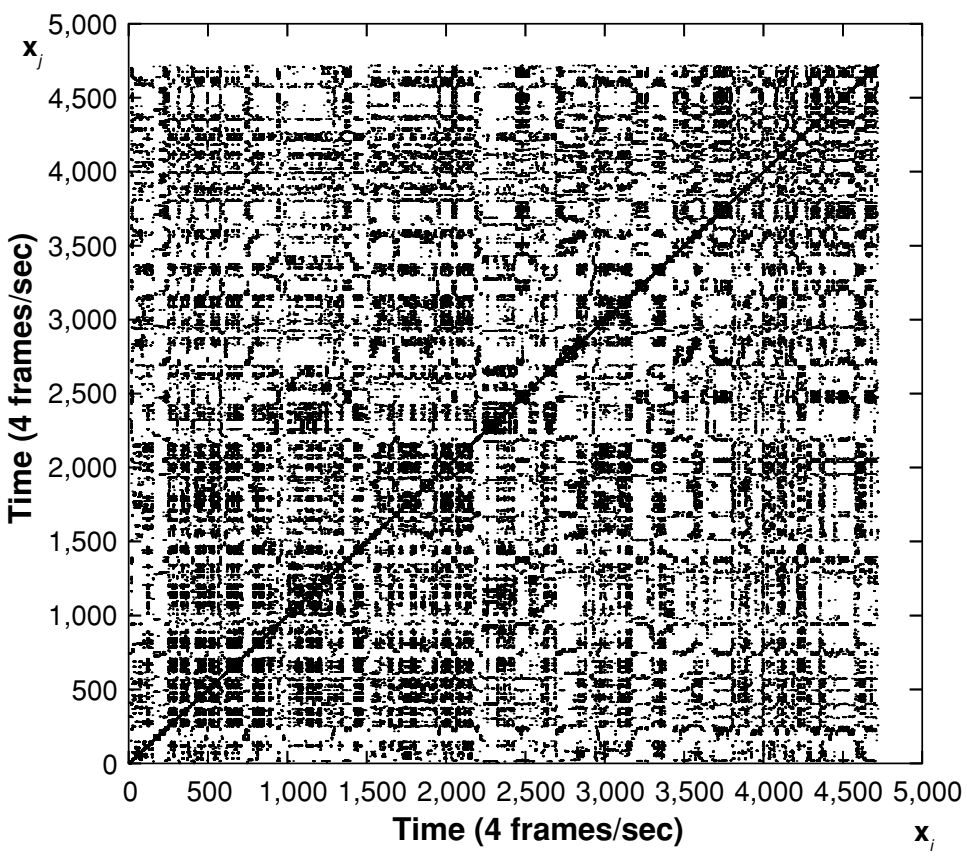

Figure 3. Recurrence plot of the integrated joint angle displacement time series with dimension $m=4$ and $\varepsilon=30.0$. The horizontal and vertical axes display the time series $x_{i}$ and $x_{j}$, respectively. 

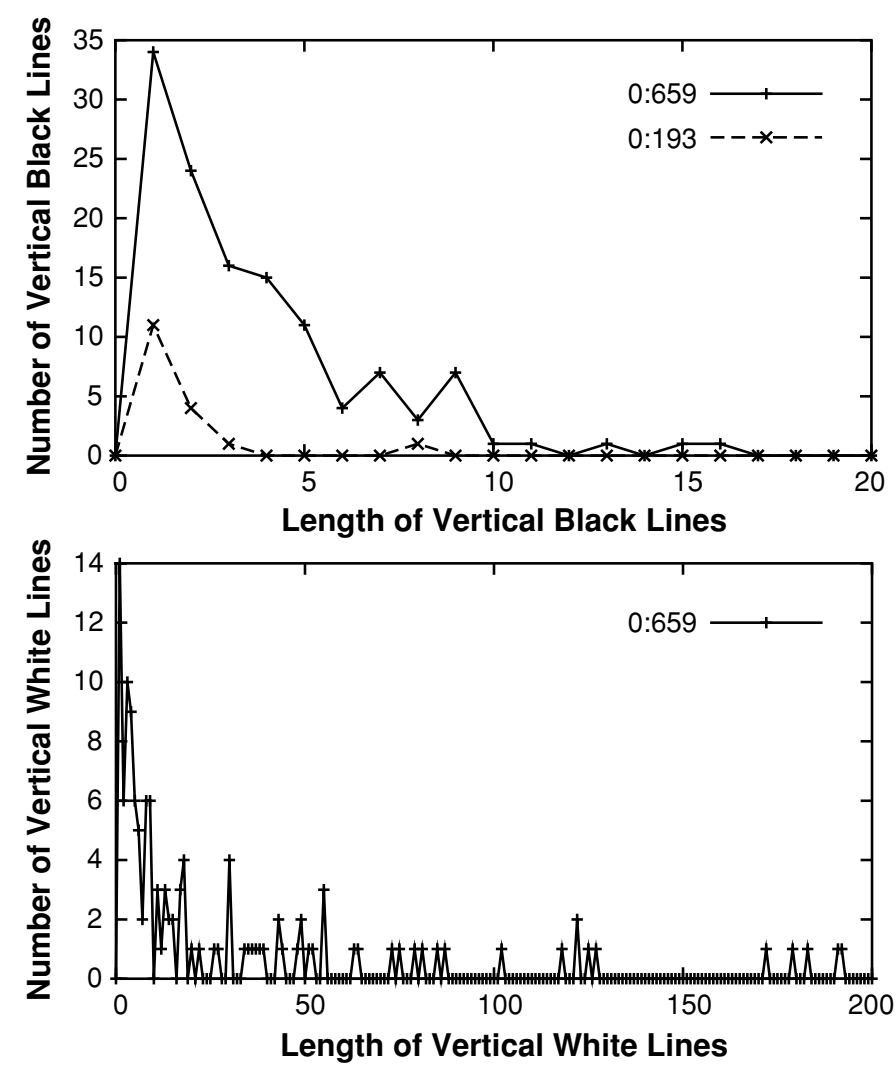

Figure 4. Frequency distribution of vertical structures in the recurrence plot. Top: The number of vertical black lines on the $y$-axis, plotted against the length of vertical black lines on the $x$-axis for Sample Points 193 and 659. Bottom: The number of vertical white lines on the $y$-axis, plotted against the length of vertical white lines on the $x$-axis for Sample Point 659.

or simultaneously, whereby they took 1 of the 16 configurations, mostly for a varying span of time. The cumulative time of staying in a given configuration, plotted against the frequency of recurring to the same configuration, shows a distribution of data points along a diagonal, which presents a high correlation between these two parameters (Figure 5). This correlation shows that those configurations that were held for the longest cumulative time were the same ones that were taken with the highest frequency. Furthermore, the plot demonstrates that there is one configuration that clearly shows the highest values.

\section{DISCUSSION}

Our analyses of motor behavior uncovered a preferred configurational position of single limbs displaying confined ranges of joint angles. Whereas the unimodal distribution of the arm positions in a lower scope of angles is biomechanically plausible, the bimodal shape of the distribution of the angles of the legs in the low and high ranges of angles appears somehow remarkable. Here, the peaks in the distributions of leg- and arm-related data can be understood as reference points in the movement patterns of limbs.
In addition, our analyses have shown that all the configurations with the longest cumulative time of staying are, at the same time, those with the highest frequency of recurrence. This phenomenon is reminiscent of the concept of home base behavior, developed by Eilam and Golani (1989) for studies of the exploratory behavior of rats placed in a novel environment. Crucial characteristics of the home base are the longest cumulative time of staying in this place and the highest number of visits. Despite the clear differences between the two accomplishments - that is, the locomotion of a rat and the spontaneous movements of a newborn - there are parallels that suggest that the special static states or configurations of newborn movements can be considered as analogous bases in which the system can settle and start new movements.

The RP of the kinematic data shows that (1) the dynamics of the system can be characterized by static states, (2) certain configurations are taken especially often in defined time windows, characterizing these time frames by certain combinations of configurations, and (3) the durations of staying and the intervals of recurrence of decisive configurations are variable. With reference to these features, the RP indicates that combinations of configurations in given time windows act as home base configurations. 


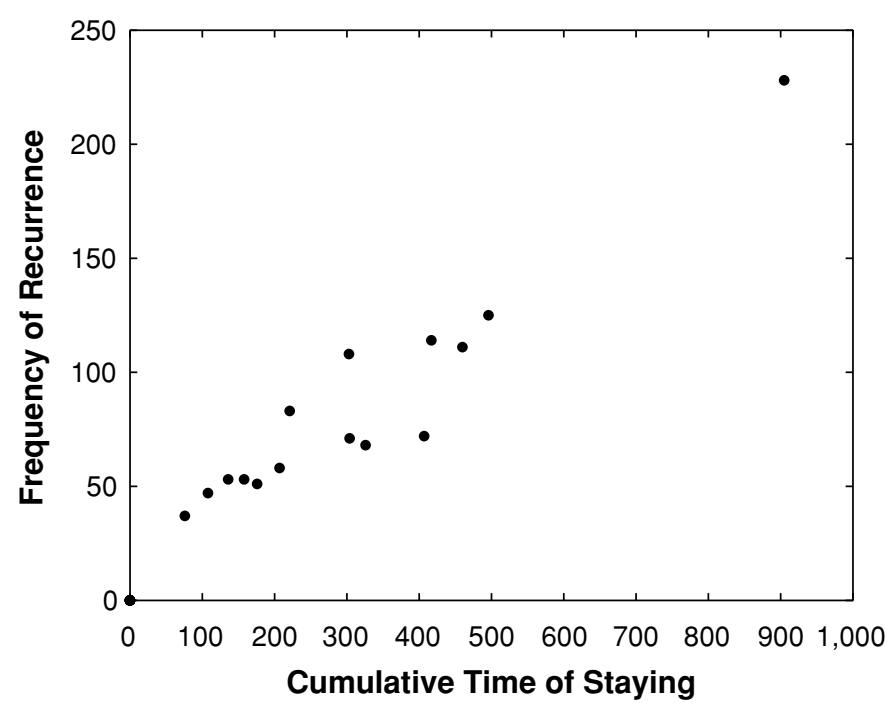

Figure 5. Correlation of the cumulative time of staying in a given configuration on the $x$-axis versus the frequency of recurrence to this configuration on the $y$-axis.

This reveals another parallel to the home base behavior of rats, which can establish several transient home bases in the course of the exploratory process (Tchernikowsky, Benjamini, \& Golani, 1995).

The similarity of RPs on different time scales suggests the existence of principles of organization that could play a role at different hierarchical levels (Anderson, 2000). On a lower level, single limbs seem to indicate reference points to which they return. On a higher level, however, the organization of configurations seems to be more complex and related to association groups that require a special reference system. We tend to presume that the combinations of reference configurations in the spontaneous movements of newborns can be considered as groups of temporally associated configurations and also that the spontaneous movements can be characterized by a structural hierarchy. Such presumption requires further inquiries into the temporal diversity of intervals within and between the various association groups, which can be tested, for example, by analyzing the length of vertical structures in the RP.

Since all individuals show one or several reference configurations, which are different for different individuals and even can change over time, the tendency to establish these reference points appears quite notable. We assume that these features do not simply reflect physiological properties or constraints of the body and, thus, tend to explain them as an endogenous component of motor behavior. Such a view would, however, require further inquiries into the reference system for static configurations and for the dynamic part of spontaneous movements as well. Future investigations could be done by analyzing the temporal parameters represented by the length of the vertical structures in the RP in reference to home base configurations.

Our finding that spontaneous movements are structured by static configurations and that newborns return to spe- cific configurations could be explained also by a mechanism that supplements the model cited above. That is, there could be some kind of memory system that captures the spatial relationship of the limbs and provides a reference frame for returning to this configuration. The mechanism that allows an animal to return to its home base is called the path integration system (Biegler \& Morris, 1996; Etienne et al., 1996), which permits position and direction to be updated solely on the basis of ideothetic information. McNaughton et al. (1996) proposed "an intrinsic, 2dimensional manifold within a high-dimensional neuronal representation space in which locations are defined by stable patterns of neural activity and in which there are orderly proximity relationships among the locations" (p. 180). The preconfiguration of a state space topology of "a two-dimensionally organized system of stable attractors would provide a robust mechanism for the spontaneous, off-line reactivation of recent experience that is thought to be necessary for memory consolidation" (McNaughton et al., 1996, p. 183). It seems rewarding to assume that newborns also may use their physical body to examine or develop such a two-dimensionally organized system of attractors. Support for this assumption can be derived from our evidence that multiple reference configurations can be coded on the basis of angular (2-D) motion signals.

Our finding of reference configurations in newborn movements is reminiscent of a theory suggesting postural coding as a general method of movement control. This concept has been particularly successful in the study of speech and facial expression (Fowler, Rubin, Remez, \& Turvey, 1980) and of limb movements (Rosenbaum, Loukopoulos, Meulenbroek, Vaughan, \& Engelbrecht, 1995). The theory has also been supported by neurological research in nonhuman primates (Niemitz, 1989). More recently, electrical stimulation of special motor cortex areas 
induced movement patterns of the related body parts from any initial configuration toward a single final posture (Graziano, Taylor, Moore, \& Cooke, 2002). The performance features of these movements are remarkably consistent with the characteristics of configurations that, in our study, were uncovered for start and target postures in the motor behavior of newborns. With this as a reference, we would like to advertise noninvasive methods, such as the methodological concept introduced here, for further applications in research into motor behavior and control.

\section{REFERENCES}

Anderson, C. M. (2000). From molecules to mindfulness. How vertically convergent fractal time fluctuations unify cognition and emotion. Consciousness \& Emotion, 1, 193-226.

Angulo-Kinzler, R. M., Ulrich, B., \& Thelen, E. (2002). Threemonth-old infants can select specific leg motor solutions. Motor Control, 6, 52-68.

Barnes, M. R., Crutchfield, C. A., \& Heriza, C. B. (1978). The neurophysiological basis of patient treatment: Vol. 11. Reflexes in motor development. Morgantown, WV: Stokesville.

Bartlett, D. (1997). Primitive reflexes and early motor development. Journal of Developmental \& Behavioral Pediatrics, 18, 151-157.

Beim Graben, P., \& Kurths, J. (2003). Detecting subthreshold events in noisy data by symbolic dynamics. Physical Review Letters, 90 , 100602.

Biegler, R., \& Morris, R. G. M. (1996). Landmark stability: Studies exploring whether the perceived stability of the environment influences spatial representation. Journal of Experimental Ecology, 199, 187-193.

Capute, A. J., Accardo, P. J., Vining, E. P. G., Rubenstein, J. E., \& Harryman, S. (1978). Primitive reflex profile. Baltimore: University Park Press.

Corbetta, D., \& Thelen, E. (1996). The developmental origins of bimanual coordination: A dynamic perspective. Journal of Experimental Psychology: Human Perception \& Performance, 22, 502-522.

Craig, C. M., \& LEE, D. N. (1999). Neonatal control of sucking pressure: Evidence for an intrinsic tau-guide. Experimental Brain Research, 124, 371-382.

Crook, C. K. (1979). The organization and control of infant sucking. Advances in Child Development \& Behavior, 14, 209-252.

Eckmann, J. P., Kamphorst, S. O., \& Ruelle, D. (1987). Recurrence plot of dynamical system. Europhysics Letters, 4, 973-977.

Eilam, D., \& Golani, I. (1988). The ontogeny of exploratory behavior in the house rat (Rattus norvegicus): The mobility gradient. Developmental Psychobiology, 21, 679-710.

EIlam, D., \& Golani, I. (1989). Homebase behavior of rats (R. norvegicus) exploring a novel environment. Behavioral Brain Research, 34, 199-211.

Etienne, A. S., Maurer, R., \& SÉguinot, V. (1996). Path integration in mammals and its interaction with visual landmarks. Journal of Experimental Biology, 199, 201-209.

Fowler, C. A., Rubin, P., Remez, R. E., \& Turvey, M. T. (1980). Implications for speech production of a general theory of action. In B. Butterworth (Ed.), Language production: Vol. 1. Speech and talk (pp. 373-420). London: Academic Press.

GolANI, I. (1992). A mobility gradient in the organization of vertebrate movement: The perception of movement through symbolic language. Behavioral \& Brain Sciences, 15, 249-308.

Graziano, M. S. A., Taylor, C. S. R., Moore, T., \& Cooke, D. F. (2002). The cortical control of movement revisited. Neuron, 36, 349-362.

HaO, B. L. (1991). Symbolic dynamics analysis of chaotic time series with a driven frequency. Physica, 51D, 161.

HOFSTEN, C. VON (1982). Eye--hand coordination in the newborn. Developmental Psychology, 18, 450-461.

Korner, A. F., \& BeAson, L. M. (1972). Association of two congenital organized behavior patterns in the newborn: Hand-mouth coordination and looking. Perceptual \& Motor Skills, 35, 115-118.

McNaughton, B. L., Barnes, C. A., Gerrard, J. L., Gothard, K., Jung, M. W., Knierim, J. J., ET AL. (1996). Deciphering the hippocampal polyglot: The hippocampus as a path integration system. Journal of Experimental Biology, 199, 173-185.

NIEMITZ, C. (1989). Erbe und Umwelt: Zur Natur von Anlage und Selbstbestimmung des Menschen (2nd ed.). Frankfurt am Main: Suhrkamp.

NiEmitz, C. (2002). Kinematics and ontogeny of locomotion in monkeys and human babies. Zeitschrift für Morphologie und Anthropologie, 83, 383-400.

Otт, E. (1993). Chaos in dynamical systems. Cambridge: Cambridge University Press.

Peiper, A. (1963). Cerebral function in infancy and childhood. New York: Consultants Bureau.

Rosenbaum, D. A., Loukopoulos, L. D., Meulenbroek, R. G., Vaughan, J., \& Engelbrecht, S. E. (1995). Planning reaches by evaluating stored postures. Psychological Review, 102, 28-67.

Takaya, R., Yukuo, K., Bos, A. F., \& Einspieler, C. (2003). Preterm to early postterm changes in the development of hand-mouth contact and other motor patterns. Early Human Development, 75, 193-202.

Tchernikowsky, O., Benjamini, Y., \& Golani, I. (1995). Constraints and the emergence of "free" exploratory behavior in rat ontogeny. Behavior, 133, 519-539.

Thelen, E., \& Fisher, M. (1983). The organization of spontaneous leg movements in newborn infants. Journal of Motor Behavior, 15, 353377.

Thelen, E., Skala, K., \& Kelso, J. A. S. (1987). The dynamic nature of early coordination: Evidence from bilateral leg movements in young infants. Developmental Psychology, 23, 179-186.

Webber, C. L., JR., \& Zbilut, J. P. (1994). Dynamical assessment of physiological systems and states using recurrence plot strategies. Journal of Applied Physiology, 76, 965-973.

(Manuscript received September 30, 2005; revision accepted for publication April 26, 2006.) 